\title{
Redtacton: A Smarter Network
}

\author{
Dushyant Chauhan
}

\begin{abstract}
RedTacton technology is an easy to use as well as convincing innovation that builds up effective communication among individuals and items associated in a closer vicinity. This paper exhibits model of a human zone organizing advancements that empowers correspondence by methods for "Contacting". This technology works in transmitting the signals through mobile terminals and such similar terminals that are implanted in the environment. In order to overcome the weak radio signals and transmit data at greater speeds efficiently, this network is introduced in the field of communication technology. The technology is based on transmitting the information signals through human body that ultimately supports "IEEE 802.3 half duplex communication". The paper focuses on the principle of utilising the electric field for Human Area Networking.
\end{abstract}

Keywords: Human area network, Red-Tacton mechanism, half-duplex communication, IEEE 802.3

\section{INTRODUCTION}

Human Area Network or Red Tacton is a technology introduction by Nippon that provides a high data speed and safe path for transmission of the signals. It is used for communication between the terminals of the mobile phones as well as other nearby objects. This technology is used so as a replacement to the communication cables[1], [2]. The transmission path is established as soon as a human body comes in contact with its nearby device and thus the communication begins. Previous technologies were focused to solve the problem of "last meter". Last meter is basically the extreme range of communication.

This limitation is overcome by photonic electric field sensors which is basically human area network called as "RedTacton".

\section{REDTACTON TECHNOLOGY}

RedTacton consists of a transmitter for inducing electric field of less intensity on the surface of the body[3].

As soon as a body comes in contact with the RedTacton trans-receiver a transmission path is said to be established. The communication is established in these paths when the terminals associated with the human body comes in contact with the terminals associated with the devices.

The acquisition of the data from these transceivers is low as the strength of the received signals is quite low. In order to overcome this limitation RedTacton uses a technique called "Electric Field Photonics." This technique uses a LASER beam that is to be passed through a crystal which is electro-optic in nature that deflects light based on the strength of the electric field surrounding it[4]. The deflected signals thus obtained are measured and then converted back into electrical signals in order to retrieve the transmitted data.

The above equation shows the generated electric field[5]. Where Ea is the electric field induced by transmitter. Eb is the electric field that returns to the ground of the transmitter. Ec is the electric field present in the receiver end. Es is the electric field that is detected at the receiver end.

\section{SALIENT FEATURES OF REDTACTON}

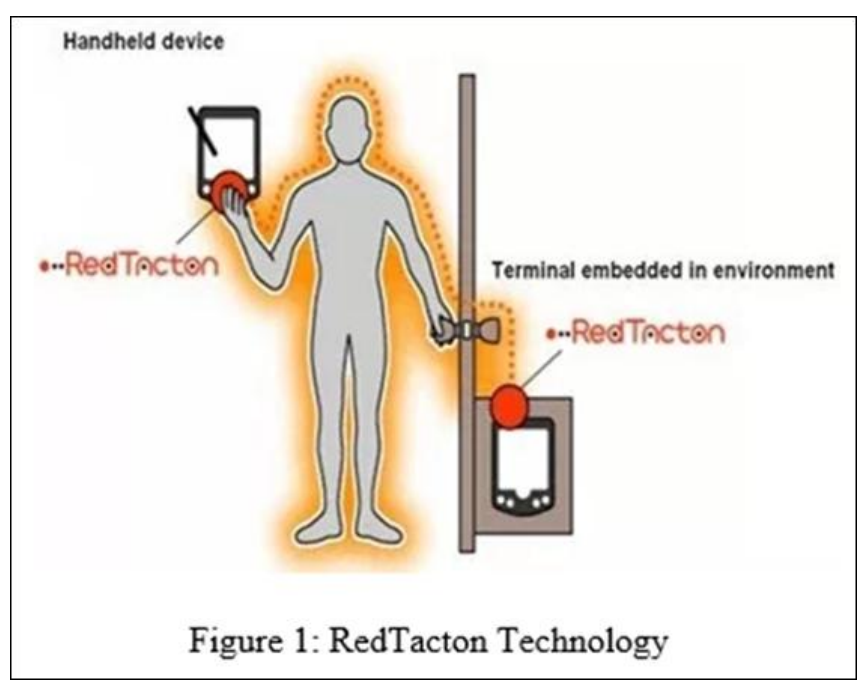

Basically RedTacton technology possess three main features.

1. Touch Feature

2. Broad band Feature

3. Multi-medium Feature

\section{Touch Feature}

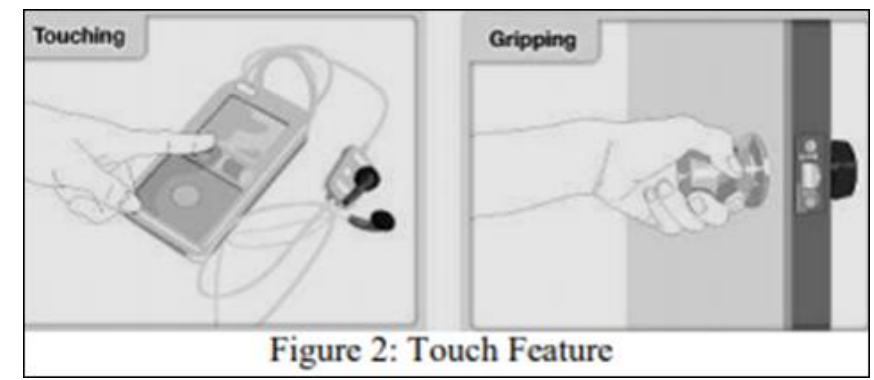

Every mode of communication can be performed with just a physical touch. These include stepping, touching, walking, gripping, sitting and so on as pulses or triggers to perform different functions related to initiation and completion of the equipment, retrieval of data as well as locking and unlocking the device[6].

\footnotetext{
Revised Manuscript Received on 14 September, 2019.

Dushyant Chauhan, Department of Computer Science and Engineering, Sanskriti University, Uttar Pradesh, India.(Email: sanpubip@gmail.com)
} 


\section{Broad Band Feature}

Data speed is the most important factor in a communication for which a system/ technology can be accepted or rejected. This system provides an ideal speed of about $10 \mathrm{Mbps}$. This technological model uses the speed of $10 \mathrm{Mbps}$ for full duplex communication.

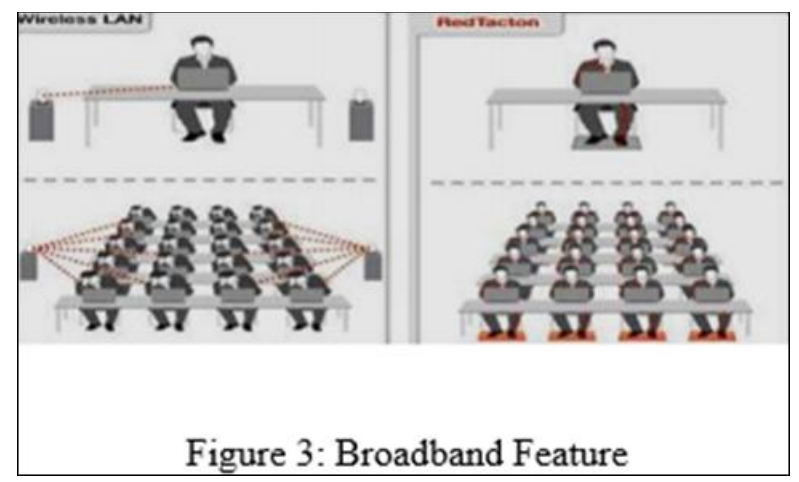

\section{Multi-medium Feature}

RedTacton utilizes a broad range of materials as a medium for transmission, as long as the conductive or dielectric material is present.

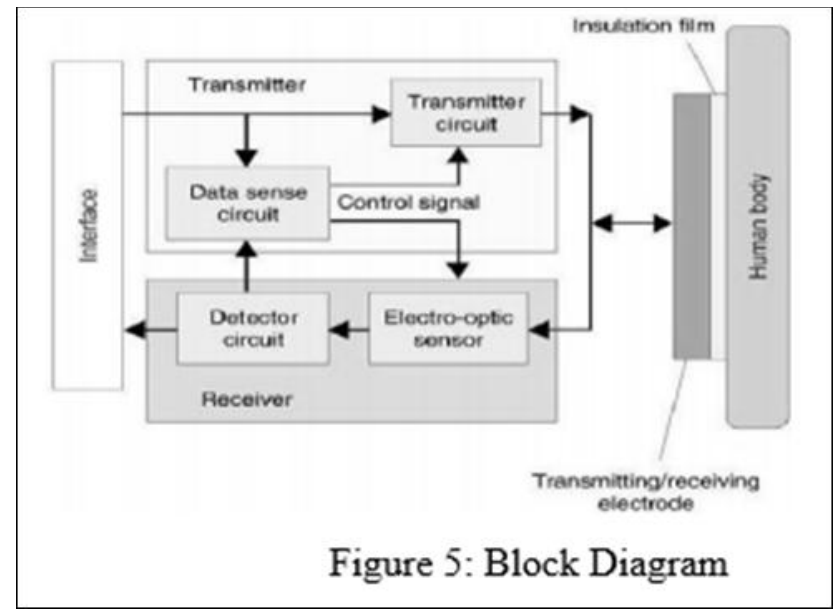

\section{WORKING PRINCIPLE}

RedTacton technology contains a transmitter as well as receiver end. When a body comes is used as medium in RedTacton transceiver, the transmission of signals is carried out. When the human body is not in contact with the terminals of the device, the transmission of data gets interrupted. The terminals are embedded in the device or are carried out by the user itself. Human parts such as arms, feet, legs, torso, fingers, clothes and shoes of a person can be used as a communicating medium[7].

The signal is sent to the data sensing circuit as well as to the transmitter circuit. The signal is sensed by the data sensing circuit and when the data is sensed, the control signals thus generated are sent to the transmitter circuit[8][11]. Varying electric field is obtained from the transmitter body. The change in electric field is obtained by the help of sensors that are electro-optic in nature.

The output obtained from these sensors is sent to the detector circuit which is further sent to the receiving end of the device.

\section{APPLICATIONS \& RESULTS}

RedTacton technology has a wide range of application in various fields.

\section{One-to-one services}

The information regarding the attributes is sent to the devices that are in touch with each other. Based on the information regarding the attributes received from the receiver appropriate service is provided to the user.

\section{Marketing Application}

This technology is also applicable in the field of marketing. In this, the customer has to stand near the advertising panel, the information that matches the individual's attributes are displayed automatically. The customer gets in depth information regarding the interested products by simply touching the products. The information is displayed on the smart device associated with the user[7], [12].

\section{Intuitive Operations}

A print out can also be generated by touching the desired printer in one hand and a PC or any other source with the other hand in order to establish a link/medium for the signals to propagate.

Transferring of songs from any audio devices to a music player in just a single touch.

\section{Exchange of data}

Authentication and encryption of the data.

Transferring group photos instantly into individual devices.

\section{Security Application}

The RedTacton devices can be installed on entrances and other security systems for secure access with just a touch. During authentication all the user details could be logged on[13]-[15].

\section{CONCLUSION}

RedTacton technology proves itself to be better when compared with similar other technologies. It has a data transmission rate of $10 \mathrm{Mbps}$ with in a particular range. This technology also emits the issue of last meter to a greater extent. It uses a human body as a communicating medium with the other electronic devices. The devices are triggered by simple touching, griping, holding or even through human clothes and shoes. This technology overcomes the limitations of the Bluetooth technology and therefore can act as a replacement in near future. It can also bring an end to the use of cables for data transmission.

\section{REFERENCES}

1. Y. Kado and M. Shinagawa, 'RedTacton near-body electric-field communications technology and its applications', NTT Tech. Rev., 2010.

2. Y. Perwej, 'A Literature Review of the Human Body as a Communication Medium using RedTacton', Commun. 
Appl. Electron., 2016.

3. S. A. Adewuyi, I. O. Aiyedun, and O. T. Balogun, 'Redtacton: Enhancing ubiquitous computing services', in Lecture Notes in Engineering and Computer Science, 2013.

4. B. V. J., 'REDTACTON-THE FORWARD THINKING OF HUMAN AREA NETWORK', Int. J. Res. Eng. Technol., 2012.

5. 'Survey on RedTacton: An Innovative Human Area Networking Technology', Int. J. Sci. Res., 2016.

6. M. S. Sarwar et al., 'Bend, stretch, and touch: Locating a finger on an actively deformed transparent sensor array', Sci. Adv., 2017.

7. M. Seyedi, B. Kibret, D. T. H. Lai, and M. Faulkner, 'A survey on intrabody communications for body area network applications', IEEE Trans. Biomed. Eng., 2013.

8. J. Xing and Y. Zhu, 'A survey on body area network', in Proceedings - 5th International Conference on Wireless Communications, Networking and Mobile Computing, WiCOM 2009, 2009.

9. H. Wang, X. Tang, C. S. Choy, and G. E. Sobelman, 'Cascaded Network Body Channel Model for Intrabody Communication', IEEE J. Biomed. Heal. Informatics, vol. 20, no. 4, pp. 1044-1052, 2016.

10. M. H. Seyedi and D. T. Huei Lai, 'Effect of limb joints and limb movement on intrabody communications for body area Network applications', J. Med. Biol. Eng., vol. 34, no. 3, pp. 276-283, 2014.

11. C. Kumar and H. R. Italia, 'Intrabody communication for human area network application', in 2014 International Conference for Convergence of Technology, I2CT 2014, 2014.

12. B. Latré, B. Braem, I. Moerman, C. Blondia, and P. Demeester, 'A survey on wireless body area networks' Wirel. Networks, 2011.

13. M. Li, W. Lou, and K. Ren, 'Data security and privacy in wireless body area networks', IEEE Wirel. Commun., 2010.

14. K. Gündoğdu and A. Çalhan, 'An Implementation of Wireless Body Area Networks for Improving Priority Data Transmission Delay', J. Med. Syst., 2016.

15. L. Zhong, D. El-Daye, B. Kaufman, N. Tobaoda, T. Mohamed, and M. Liebschner, 'OsteoConduct: Wireless body-area communication based on bone conduction', in BODYNETS 2007 - 2nd International ICST Conference on Body Area Networks, 2007. 